\title{
Adaptive Backstepping Sliding Mode Control for Direct Driven Hydraulics
}

\author{
Shuzhong Zhang ${ }^{1, *}$, Tianyi Chen ${ }^{1}$, Fuquan Dai ${ }^{2}$ \\ 1 School of Mechanical and Automotive Engineering, Fujian University of Technology, Fuzhou, China; \\ shuzhong_zhang@outlook.com; chen_tian_yi@outlook.com \\ 2 Fujian Haiyuan Composite Materials Technology Co., Ltd \\ * Correspondence: shuzhong_zhang@outlook.com; Tel.: +86-591-228-63232
}

\begin{abstract}
Due to the advantages of high energy efficiency and environmental friendliness, the electro-hydraulic actuator (EHA) plays a vital role in fluid power control. One variant of EHA, double pump direct driven hydraulics (DDH), was proposed, which consists of double fixeddisplacement pumps, a servo motor, an asymmetric cylinder, and auxiliary components. This paper proposed an adaptive backstepping sliding mode control (ABSMC) strategy for DDH to eliminate the adverse effect produced by parametric uncertainty, nonlinear characteristics and the uncertain external disturbance. Based on theoretical analysis, the nonlinear system model was built and transformed. Further, by defining the sliding manifold and selecting a proper Lyapunov function, the nesting problems (of the designed variable and adaptive law) caused by uncertain coefficients were solved. Moreover, the adaptive backstepping control and the sliding mode control were combined to boost system robustness. At the same time, the controller parameter adaptive law is derived from Lyapunov analysis to guarantee the stability of the system. Simulations of the DDH were performed with the proposed control strategy and proportional-integral-differential (PID), respectively. The results show that the proposed control strategy can achieve better position tracking and stronger robustness in parameter changing compared with PID.
\end{abstract}

Keywords: Adaptive backstepping; Sliding mode control; Electro- hydraulic actuator (EHA); Direct driven hydraulics (DDH); Position tracking; proportional-integral-differential (PID).

\section{Introduction}

The hydraulic system is widely used in robots, automobiles, aerospace and defence industries due to its advantages, such as fast response, high force and power density, reliability, and robustness [1]. The system can be divided into two categories: the valve-controlled system and the pump-controlled system. Owning to the high accuracy and low-cost, the valve-controlled system is adopted more. How ever, with the energy crisis and pollution issue, the pump-controlled system has attracted rising attention because of higher energy efficiency. In terms of energy, the pump-controlled system eliminates significant throttling loss, which accounts for $44 \%$ of the energy loss of the valvecontrolled system [2,3].

One division of pump-controlled system, the electro-hydraulic actuator (EHA), is usually referred to a compact and reliable self-contained unit composed of the electric motor, pump/motors, hydraulic cylinder and auxiliary components. EHA can be divided into three classifications: (1) fixed 
displacement pump and variable speed electric motor, (2)variable displacement pump and fixed speed motor, and (3) variable displacement pump and variable speed electric motor. The third configuration can provide the highest energy efficiency, but it costs more and requires more complex control systems to achieve maximum efficiency [4,5]. Compared to the other two classifications, the first scheme has a slow dynamic response, but it has the properties of low -cost, simplicity, and highefficiency [5-8]. Hence, it attracts more and more attention. How ever, there are strong nonlinearities and uncertainties in EHA, such as nonlinear friction, parameter uncertainty and unknown external disturbances. Therefore, the controller design of the EHA faces significant challenges [9].

Due to the uncertainty and disturbance in the whole system, the control performance can't be guaranteed by applying the proportional-integral-derivative (PID) control method [10]. Hence, many studies related to the position control of the EHA are conducted. Among them, some control strategies have achieved high-performance in position control, including adaptive control [11, 12], sliding mode variable structure control [13, 14], fuzzy control [15] and neural network PID [16]. Furthermore, then in order to solve the problem of load disturbance, nonlinear and parameter uncertainty in the position control of closed pump control system, the design of a fuzzy logic controller [17], the design of robust model predictive controller [18] and sliding mode control [19] are studied. Although the sliding mode control can reduce the influence of parameters and complex changes on the system, the sliding mode control has significant jitter, and the design process of the control system is relatively complex. Sliding mode variable structure control requires higher switching gain and stronger chattering. Adaptive backstepping control can improve the control performance of nonlinear systems.

The pump-controlled system can also be divided into pump-controlled symmetric cylinder and pump-controlled asymmetric cylinder. Among them, the pump-controlled symmetric cylinder technology started earlier and has been mature. How ever, the asymmetry of the flow between the two chambers of the differential cylinder becomes the primary problem that must be solved to realize the pump controlled differential cylinder technology. References $[5,20]$ proposed an evolutionary form of EHA, double-pump direct driven hydraulics (DDH), which can solve the problem of hydraulic cylinder flow mismatch. In order to improve the position control accuracy of the doublepump DDH, an adaptive backstepping slide mode control (ABSMC) method is proposed. The proposed method uses the backstepping design to get a sliding mode controller which guarantees the stability of the control system. The controller requires neither the accurate system model parameters nor uncertainty boundary of the uncertain parameters. The ABSMC uses a simplified linearized model with unknown parameters, which is superior to the existing sliding mode controller.

In this paper, sections 2 introduces the DDH system, sections 3 creates the linear math ematical model, sections 4 gives the design procedure of the ABSMC controller and proves the stability of it, sections 5 gives the simulation and analysis, and sections 6 draws the conclusions.

\section{DDH and Modelling}

\section{1. $D D H$}

Double-pump DDH uses double fixed displacement pump driven by one variable speed electric motor to control a differential cylinder [5]. The schematics of the DDH system is shown in Figure 1. Parameters of the main components in the simulation are shown in Table1 [5]. 


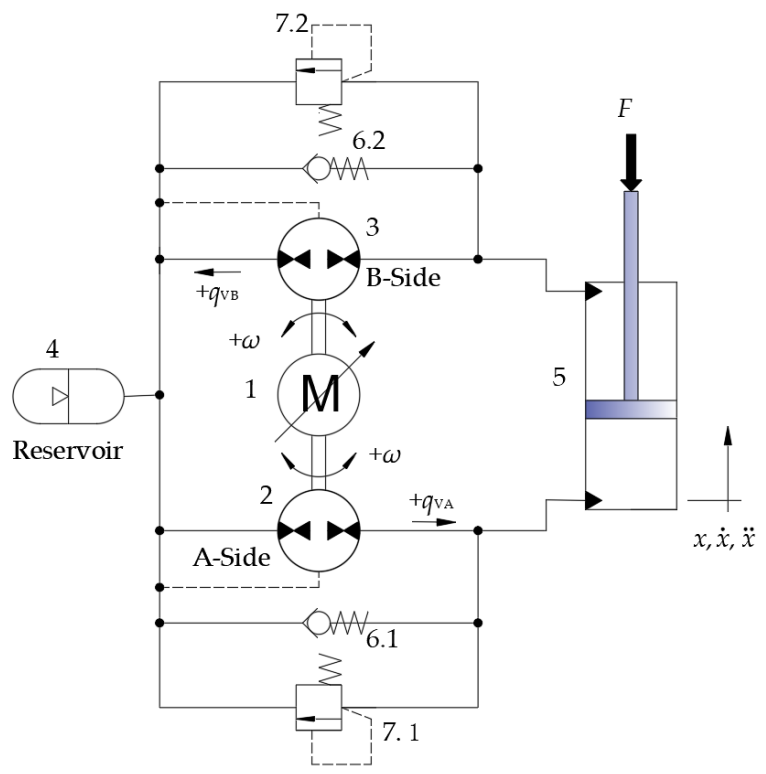

Figure 1. Schematics of DDH. 1: Servo motor, 2: A-side pump, 3: B-side pump, 4: Hydraulic accumula tor, 5: Cylinder, 6: Checkvalve, 7: Relief valve

Table 1. Main parameters of the components

\begin{tabular}{|c|c|c|c|}
\hline NO. & Component & Parameters & Value \\
\hline \multirow{2}{*}{1} & Synchronous Torque & Rated Torque [Nm] & 4.5 \\
\hline & Motor & Rated Speed [rpm] & 2500 \\
\hline 2 & A-Side Pump & $\begin{array}{l}\text { Volumetric Displacement }\left(D_{\mathrm{A}}\right) \\
{\left[\mathrm{cm}^{3} / \mathrm{rev}\right]}\end{array}$ & 13.03 \\
\hline 3 & B-Side Pump & $\begin{array}{l}\text { Volumetric Displacement }\left(D_{\mathrm{B}}\right) \\
{\left[\mathrm{cm}^{3} / \mathrm{rev}\right]}\end{array}$ & 9.35 \\
\hline 4 & $\begin{array}{l}\text { Hydraulic } \\
\text { Accumulator }\end{array}$ & Volume [L] & 0.7 \\
\hline 5 & Cylinder & Dimensions [mm] & $60 / 30 * 400$ \\
\hline
\end{tabular}

\subsection{Modelling of $D D H$}

This section describes the mathematical model of DDH. Compared to the pump-controlled system, the frequency of the electric motor is much higher. Hence, the dynamics of the electric motor is omitted.

The hydraulic cylinder flow continuity equation can be expressed by:

$$
\begin{gathered}
q_{\mathrm{A}}=A_{\mathrm{A}} \dot{x}+c_{\mathrm{i}}\left(p_{\mathrm{A}}-p_{\mathrm{B}}\right)+c_{\mathrm{e}} p_{\mathrm{A}}+\frac{V_{\mathrm{A}}}{\beta_{e}} \dot{p}_{\mathrm{A}}, \\
q_{\mathrm{B}}=-A_{\mathrm{B}} \dot{x}+c_{\mathrm{i}}\left(p_{\mathrm{A}}-p_{\mathrm{B}}\right)-c_{\mathrm{e}} p_{\mathrm{B}}-\frac{V_{\mathrm{B}}}{\beta_{e}} \dot{p}_{\mathrm{B}}, \\
V_{\mathrm{A}}=V_{0 \mathrm{~A}}+A_{\mathrm{A}} x, \\
V_{\mathrm{B}}=V_{0 \mathrm{~B}}-A_{\mathrm{B}} x,
\end{gathered}
$$


where $q_{\mathrm{A}}$ and $q_{\mathrm{B}}$ are the flow rates into $\mathrm{A}$ and $\mathrm{B}$ chambers, $A_{\mathrm{A}}$ and $A_{\mathrm{B}}$ the effective cross-section areas of piston side and rod side, $x$ the current position, $c_{i}$ and $c_{e}$ the internal and external leakage coefficients, $V_{\mathrm{A}}$ and $V_{\mathrm{B}}$ are the total volumes of the chamber $\mathrm{A}$ and chamber $\mathrm{B}, p_{\mathrm{A}}$ and $p_{\mathrm{B}}$ are the pressures of the chamber $A$ and chamber $B, \beta_{e}$ is the effective bulk modulus. $V_{0 \mathrm{~A}}$ and $V_{\mathrm{OB}}$ are the dead volumes of $\mathrm{A}$ and $\mathrm{B}$ chambers.

The output flow of the pump is

$$
\begin{aligned}
& q_{\mathrm{VA}}=n D_{\mathrm{A}} \eta_{\mathrm{A}}, \\
& q_{\mathrm{VA}}=n D_{\mathrm{A}} \eta_{\mathrm{A}},
\end{aligned}
$$

where $q$ VA and $q$ в are the flow rates of pump $\mathrm{A}$ and $\mathrm{B}, n$ is the motor speed; $D_{\mathrm{A}}$ and $D_{\mathrm{B}}$ are the flow rates of A-side and B-side pumps; $\eta_{\mathrm{A}}$ and $\eta_{\mathrm{B}}$ represent the efficiency of pump A and B.

The force balance equation of the piston is

$$
p_{\mathrm{A}} A_{\mathrm{A}}-p_{\mathrm{B}} A_{\mathrm{B}}=M \ddot{x}+B \dot{x}+k x+F,
$$

where $M$ is the total mass of piston and load; $F$ is the load force and disturbance acting on the piston; $B$ viscous damping coefficient of the piston; $k$ is the spring stiffness coefficient.

For the convenience of calculation, the leakage part of the hydraulic cylinder is classified as disturbance and $k=0$, define the state vectors as $\boldsymbol{x}_{0}=\left[x_{1}, x_{2}, x_{3}\right]^{T}=[x, \dot{x}, \ddot{x}]^{T}$.

The state space equation can be obtained from the double-pump DDH model:

$$
\begin{gathered}
\left\{\begin{array}{c}
\dot{x}_{1}=x_{2} \\
\dot{x}_{2}=x_{3} \\
\dot{x}_{3}=r_{1} x_{3}+r_{2} x_{2}+b_{0} u+f
\end{array}\right. \\
r_{1}=-\frac{B}{M}, r_{2}=-\frac{\beta_{e}}{M}\left(\frac{A_{\mathrm{A}}{ }^{2}}{V_{\mathrm{A}}}-\frac{A_{\mathrm{B}}{ }^{2}}{V_{\mathrm{B}}}\right), b_{0}=\frac{\beta_{e}}{M}\left(\frac{A_{\mathrm{A}} D_{\mathrm{A}} \eta_{\mathrm{A}}}{V_{\mathrm{A}}}-\frac{A_{\mathrm{B}} D_{\mathrm{B}} \eta_{\mathrm{B}}}{V_{\mathrm{B}}}\right), f=-\frac{\dot{F}}{M},
\end{gathered}
$$

where $u$ is the DDH system input, $\eta_{\mathrm{A}}=\eta_{\mathrm{B}}=85 \%$.

According to the reference [5], mechanical parameters were shown as follows: $M=100 \mathrm{~kg}$, $\beta_{e}=1.4 \times 10^{9}(\mathrm{~Pa}), \quad B=100 \mathrm{~N} \cdot \mathrm{s} / \mathrm{m}$, hydraulic cylinder parameters $V_{0 \mathrm{~A}}=55.8 \times 10^{-6} \mathrm{~m}^{3}, \quad V_{0 \mathrm{~B}}=18.2 \times 10^{-6} \mathrm{~m}^{3}$.

In actual conditions, there may be certain uncertainties in the load mass, leakage coefficient, bulk modulus, spring elastic coefficient, external load force, etc. The aim of this study is to design the controller to obtain an accurate position tracking under the condition of uncertain parameters being constant or slow time-varying.

\section{Design of ABSMC controller}

Backstepping design method, usually combined with Lyapunov-type adaptive law, comprehensively considers control law and adaptivelaw, so that the entire closed-loop system meets the expected dynamic and static performance indicators.

A single backstepping control law requires accurate modelling information of the controlled object and unable to overcome the disturbance. How ever, the ABSMC requires neither the exact system model parameters nor certainty boundary of the uncertain parameters.

According to the system simplified model in sections 3, the DDH system can be expressed as a third-order linear system. Therefore, an adaptive controller is designed for the third-order linear system. The third-order linear system can be expressed as follows:

$$
\left\{\begin{array}{c}
\dot{x}_{1}=x_{2} \\
\dot{x}_{2}=x_{3} \\
\dot{x}_{3}=a_{1} x_{1}+a_{2} x_{2}+a_{3} x_{3}+b u+d
\end{array} .\right.
$$

where, $a_{1}, a_{2}, a_{3}$ and $b$ are the unknown parameters, and $d$ is an unknown disturbance. 
The flow chart of the ABSMC is shown in Figure 2.

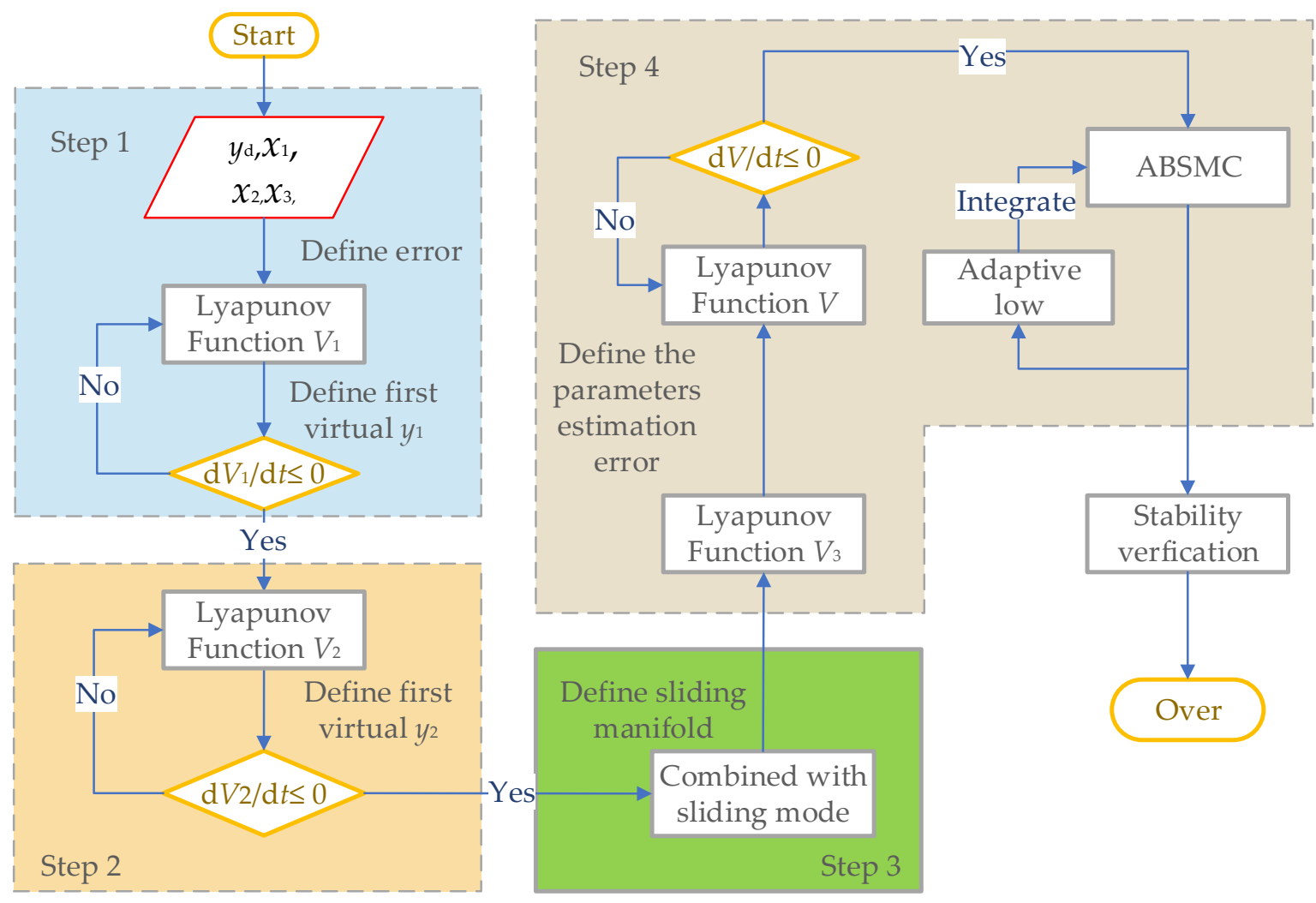

Figure 2. The flowchart of the ABSMC

\subsection{Design of ABSMC and the adaptive law of unknown parameters}

The ABSMC is designed for the system, and the adaptive law of unknown parameters is given by Lyapunov stability theorem, including the following steps.

Step 1: define the position tracking error.

All errors are defined as

$$
\left\{\begin{array}{l}
e_{1}=x_{1}-y_{\mathrm{d}} \\
e_{2}=x_{2}-y_{1} . \\
e_{3}=x_{3}-y_{2}
\end{array}\right.
$$

where, $y_{\mathrm{d}}$ is the desired position, $y_{1}$ and $y_{2}$ are virtual control variables.

The derivative of equation (10) gives

$$
\left\{\begin{array}{c}
\dot{e}_{1}=e_{2}+y_{1}-\dot{y}_{\mathrm{d}} \\
\dot{e}_{2}=e_{2}+y_{2}-\dot{y}_{1} \\
\dot{e}_{3}=a_{1} x_{1}+a_{2} x_{2}+a_{3} x_{3}+b u+d-\dot{y}_{2}
\end{array} .\right.
$$

The Lyapunov function is chosen as

$$
V_{1}=\frac{1}{2} e_{1}^{2}
$$

The derivative of equation (12) gives

$$
\dot{V}_{1}=e_{1} \dot{e}_{1}=e_{1}\left(e_{2}+y_{1}-\dot{y}_{\mathrm{d}}\right) .
$$

The first virtual control is as follows

$$
y_{1}=-k_{1} e_{1}+\dot{y}_{\mathrm{d}},
$$

where $k_{1}>0$ is a design parameter.

Substituting equation (14) into equation (13) obtains 


$$
\dot{V}_{1}=-k_{1} e_{1}{ }^{2}+e_{1} e_{2}
$$

If $e_{2}=0$, then there have $\dot{V}_{1} \leq 0$. Therefore, the backstepping algorithm is used again for the next step design.

Step 2: the Lyapunov function is chosen as

$$
V_{2}=V_{1}+\frac{1}{2} e_{2}^{2} .
$$

The derivative of equation (16) gives

$$
\dot{V}_{2}=\dot{V}_{1}+e_{2} \dot{e}_{2}=-k_{1} e_{1}^{2}+e_{1} e_{2}+e_{2}\left(e_{3}+y_{2}-\dot{y}_{1}\right) .
$$

The second virtual control is as follows

$$
y_{2}=-k_{2} e_{2}+\dot{y}_{1}-e_{1}
$$

where $k_{1}>0$ is a design parameter.

Substituting equation (18) into equation (17) obtains

$$
\dot{V}_{2}=-k_{1} e_{1}{ }^{2}-k_{2} e_{2}{ }^{2}+e_{2} e_{3} .
$$

If $e_{3}=0$, then there have $\dot{V}_{2} \leq 0$. Therefore, the first two subsystems are stable.

Step 3: Combined with sliding model control.

Sliding mode control is used and the sliding manifold is defined as

$$
s=c_{1} e_{1}+c_{2} e_{2}+e_{3}
$$

where, $c_{1}$ and $c_{2}$ are the normal number

The derivative of equation (20) gives

$$
\dot{s}_{2}=c_{1} \dot{e}_{1}+c_{2} \dot{e}_{2}+\dot{e}_{3}=c_{1}\left(x_{2}-\dot{y}_{d}\right)+c_{2}\left(x_{3}-\dot{y}_{1}\right)+a_{1} x_{1}+a_{2} x_{2}+a_{3} x_{3}+b u+d-\dot{y}_{2}
$$

Step 4: In order to avoid including the control variable $u$ in the parameter adaptive law $\hat{\tau}_{4}$ designed below, that is, to avoid loop nesting, the Lyapunov function is chosen as

$$
V_{3}=V_{2}+\frac{1}{2 b} s^{2} \geq 0
$$

The derivative of equation (22) gives

$$
\dot{V}_{3}=-k_{1} e_{1}^{2}-k_{2} e_{2}^{2}+e_{2} e_{3}+s\left[\frac{c_{1}}{b}\left(x_{2}-\dot{y}_{\mathrm{d}}\right)+\frac{c_{2}}{b}\left(x_{3}-\dot{y}_{1}\right)\right]+s\left[\frac{a_{1}}{b} x_{1}+\frac{c_{2}}{b} x_{2}+\frac{c_{2}}{b} x_{3}+u+\frac{d}{b}-\frac{1}{b} \dot{y}_{2}\right] \text {. }
$$

Define $\tau_{1}=\frac{a_{1}}{b}, \tau_{2}=\frac{a_{2}}{b}, \tau_{3}=\frac{a_{3}}{b}, \tau_{4}=\frac{d}{b}$, equation (23) can be simplified as

$$
\dot{V}_{3}=-k_{1} e_{1}{ }^{2}-k_{2} e_{2}{ }^{2}+e_{2} e_{3}+s\left[\frac{c_{1}}{b}\left(x_{2}-\dot{y}_{\mathrm{d}}\right)+\frac{c_{2}}{b}\left(x_{3}-\dot{y}_{1}\right)+\tau_{1} x_{1}+\tau_{2} x_{2}+\tau_{3} x_{3}+u+\tau_{4}-\frac{1}{b} \dot{y}_{2}\right] \text {. }
$$

Define $\tilde{\tau}_{1}=\tau_{1}-\hat{\tau}_{1}, \tilde{\tau}_{2}=\tau_{2}-\hat{\tau}_{2}, \tilde{\tau}_{3}=\tau_{3}-\hat{\tau}_{3}, \tilde{\tau}_{4}=\tau_{4}-\hat{\tau}_{4}, \tilde{a}_{4}=a_{4}-\hat{a}_{4}$ where, $\hat{\tau}_{1}, \hat{\tau}_{2}, \hat{\tau}_{3}, \hat{\tau}_{4}$ and $\hat{a}_{4}$ are the estimated values of $\tau_{1}, \tau_{2}, \tau_{3}, \tau_{4}$ and $a_{4} . \tilde{\tau}_{1}, \tilde{\tau}_{2}, \tilde{\tau}_{3}, \tilde{\tau}_{4}$ and $\tilde{a}_{4}$ are the parameters estimation errors.

The Lyapunov function is chosen as

$$
V=V_{3}+\frac{1}{2} \lambda_{1} \tilde{\tau}_{1}^{2}+\frac{1}{2} \lambda_{2} \tilde{\tau}_{2}^{2}+\frac{1}{2} \lambda_{3} \tilde{\tau}_{3}^{2}+\frac{1}{2} \lambda_{4} \tilde{\tau}_{4}^{2}+\frac{1}{2} \lambda_{5} \tilde{a}_{4}^{2} \geq 0,
$$

where $\lambda_{\mathrm{i}}>0(i=1,2,3,4,5)$ is adaptive gain.

The derivative of equation (25) gives 
$\dot{V}=-k_{1} e_{1}{ }^{2}-k_{2} e_{2}{ }^{2}+e_{2} e_{3}+s\left[c_{1} a_{4}\left(x_{2}-\dot{y}_{\mathrm{d}}\right)+c_{2} a_{4}\left(x_{3}-\dot{y}_{1}\right)\right]+s\left[\tau_{1} x_{1}+\tau_{2} x_{2}+\tau_{3} x_{3}+u+\tau_{4}-\right.$

$\left.a_{4} \dot{y}_{2}\right]+\lambda_{1} \tilde{\tau}_{1}\left(-\hat{\tau}_{1}\right)+\lambda_{2} \tilde{\tau}_{2}\left(-\hat{\tau}_{2}\right)+\lambda_{3} \tilde{\tau}_{3}\left(-\hat{\tau}_{3}\right)+\lambda_{4} \tilde{\tau}_{4}\left(-\hat{\tau}_{4}\right)+\lambda_{5} \tilde{a}_{4}\left(-\dot{\hat{a}}_{4}\right)$.

The ABSMC is designed as follows

$$
u=-c_{1} \hat{a}_{4}\left(x_{2}-\dot{y}_{\mathrm{d}}\right)-c_{2} \hat{a}_{4}\left(x_{3}-\dot{y}_{1}\right)-\hat{\tau}_{1} x_{1}-\hat{\tau}_{2} x_{2}-\hat{\tau}_{3} x_{3}-\hat{\tau}_{4}+\hat{a}_{4} \dot{y}_{2}-h_{1} s-h_{2} \operatorname{sgn}(s),
$$

where, $h_{1}$ and $h_{2}$ are design parameters.

Substituting equation (27) into equation (26) obtains

$\dot{V}=-k_{1} e_{1}{ }^{2}-k_{2} e_{2}{ }^{2}+e_{2} e_{3}-h_{2} s^{2}-h_{2} \operatorname{sgn}(s)_{s}+\tilde{\tau}_{1}\left(s x_{1}-\lambda_{1} \hat{\tau}_{1}\right)+\tilde{\tau}_{2}\left(s x_{2}-\lambda_{2} \hat{\tau}_{2}\right)+\tilde{\tau}_{3}\left(s x_{3}-\lambda_{3} \hat{\tau}_{3}\right)+$ $\tilde{\tau}_{4}\left(s-\lambda_{4} \hat{\tau}_{4}\right)+\tilde{a}_{4}\left[c_{1} s\left(x_{2}-\dot{y}_{\mathrm{d}}\right)+c_{2} s\left(x_{3}-\dot{y}_{1}\right)-s \dot{y}_{2}-\lambda_{5} \dot{\hat{a}}_{4}\right]$.

The adaptivelaw of parameter variation is

$$
\left\{\begin{array}{c}
\hat{\tau}_{1}=\frac{1}{\lambda_{1}} s x_{1}, \hat{\tau}_{2}=\frac{1}{\lambda_{2}} s x_{2}, \hat{\tau}_{3}=\frac{1}{\lambda_{3}} s x_{3}, \hat{\tau}_{4}=\frac{1}{\lambda_{4}} s \\
\dot{\hat{a}}_{4}=\frac{1}{\lambda_{5}} s\left(c_{1}\left(x_{2}-\dot{y}_{\mathrm{d}}\right)+c_{2}\left(x_{3}-\dot{y}_{1}\right)-\dot{y}_{2}\right)
\end{array} .\right.
$$

\subsection{Stability verification}

The stability condition of the system can be obtained by analyzing the Lyapunov function.

The derivative of the Lapunov function of the system.

$$
\dot{V}=-k_{1} e_{1}^{2}-k_{2} e_{2}^{2}+e_{2} e_{3}-h_{1} s^{2}-h_{2}|s|=-\boldsymbol{E}^{T} \boldsymbol{Q E}-h_{2}|s|,
$$

where,

$$
\boldsymbol{Q}=\left[\begin{array}{ccc}
h_{1} c_{1}^{2}+k_{1} & h_{1} c_{1} c_{2} & e_{2} c_{1} \\
h_{1} c_{1} c_{2} & h_{1} c_{2}^{2}+k_{2} & h_{1} c_{2}-\frac{1}{2} \\
h_{1} c_{1} & h_{1} c_{2}-\frac{1}{2} & h_{1}
\end{array}\right] .
$$

For the DDH system, the controller (27) is designed, and its parameter adaptive law (29) is obtained. If appropriate controller parameters are selected, that should satisfy the following inequality

$$
\left\{\begin{array}{c}
k_{1}>0, k_{2}>0, h_{1}>0, h_{2}>0, c_{1}>0, c_{2}>0 \\
k_{1} k_{2}+k_{1} h_{1} c_{2}^{2}+k_{2} h_{1} c_{1}^{2}>0 \\
k_{1} k_{2} h_{1}+k_{1} h_{1} c_{2}-\left(k_{1}+h_{1} c_{1}{ }^{2}\right) / 4>0
\end{array} .\right.
$$

Then $Q$ is a positive definite matrix, and then

$$
\dot{V}=-\boldsymbol{E}^{T} \boldsymbol{Q} \boldsymbol{E}-h_{2}|s| \leq 0 .
$$

Define $\boldsymbol{G}=\boldsymbol{E}^{T} \boldsymbol{Q} \boldsymbol{E}$, that

$$
\begin{gathered}
\dot{V}=-\boldsymbol{E}^{T} \boldsymbol{Q} \boldsymbol{E}-h_{2}|s| \leq-G . \\
\lim _{t \rightarrow \infty} \int_{0}^{t} \boldsymbol{G} d t \leq\left[V\left(e_{1}(0), e_{2}(0), e_{3}(0)\right)-V\left(e_{1}(\infty), e_{2}(\infty), e_{3}(\infty)\right)\right] .
\end{gathered}
$$

Then the position tracking error of the system is convergent, and the whole system is asymptotically stable.

According to Barbalat's theorem,

$$
\lim _{t \rightarrow \infty} \boldsymbol{G}=0 .
$$

Thus, it can be obtained that:

$$
\lim _{t \rightarrow \infty} e_{\mathrm{i}}=0(i=1,2,3) .
$$


The position tracking error of the system is convergent. Similarly, it can be obtained that

$$
\lim _{t \rightarrow \infty} s=0
$$

Therefore, it can be concluded that the entire hydraulic position servo system is asymptotically stable, tends to the sliding surface within a limited period and moves along the desired trajectory.

\section{Simulation and Analysis}

\subsection{Simulation model}

The theory from the preceding sections in this chapter is used for creating a Matlab/Simulink model of DDH system, as shown in Figure 3.

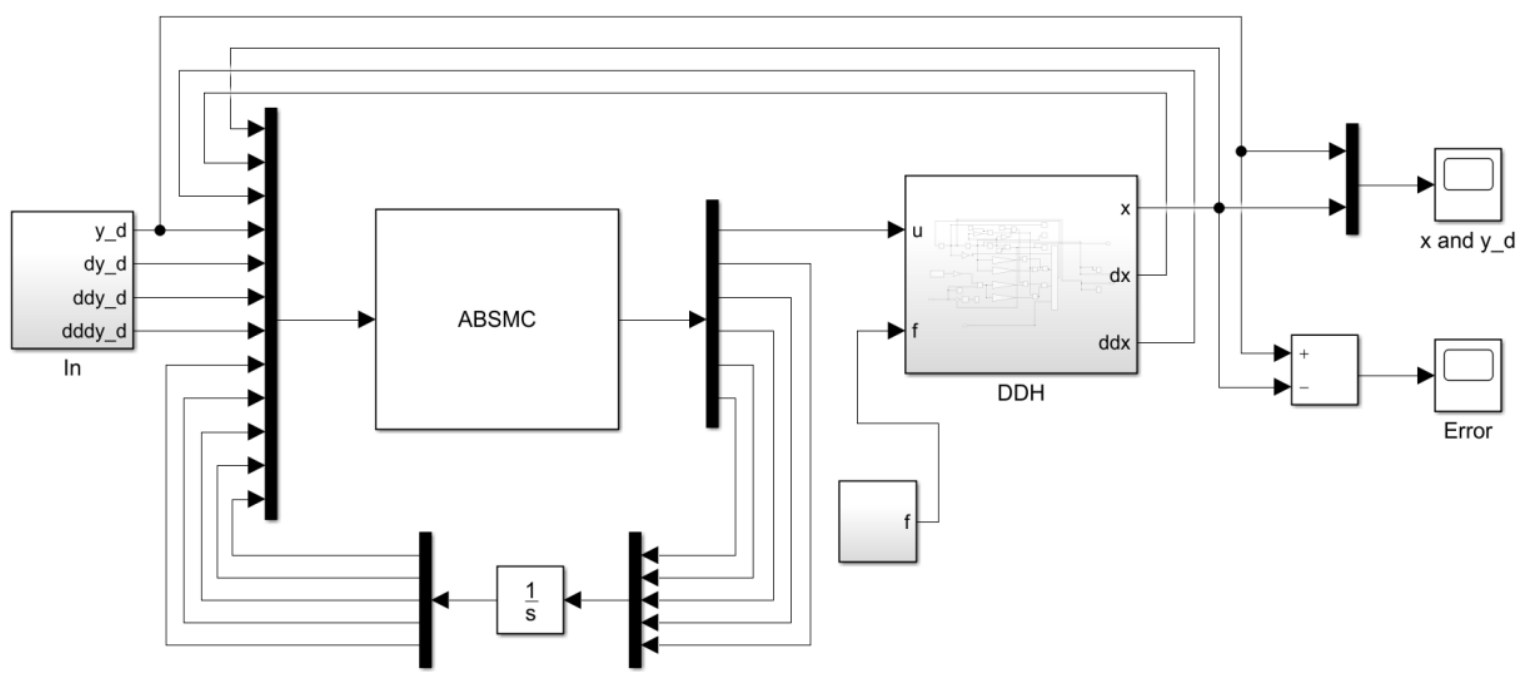

Figure 3. The schematic diagram of the Simulation model of the DDH control system

\subsection{Load and disturbance}

In order to detect the anti-disturbance ability of the controller, the sum of the load and disturbance force is set as $F_{1}=10,000-4000 \cos (5 t)$, as shown in Figure $4(a) . F_{2}$ is a triangular signal, as shown in Figure 4(b).

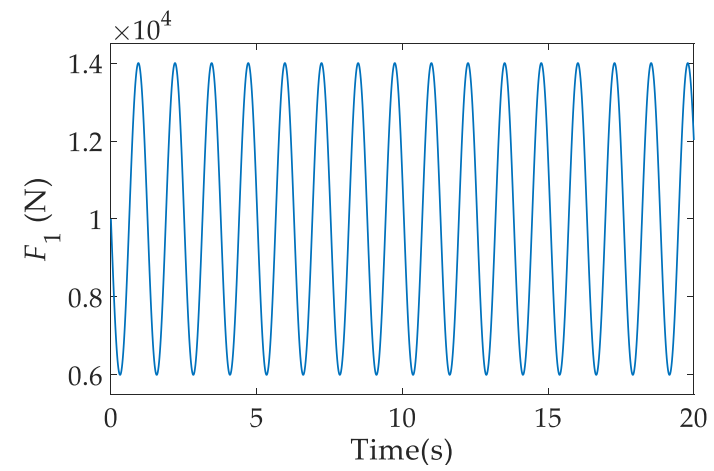

(a)

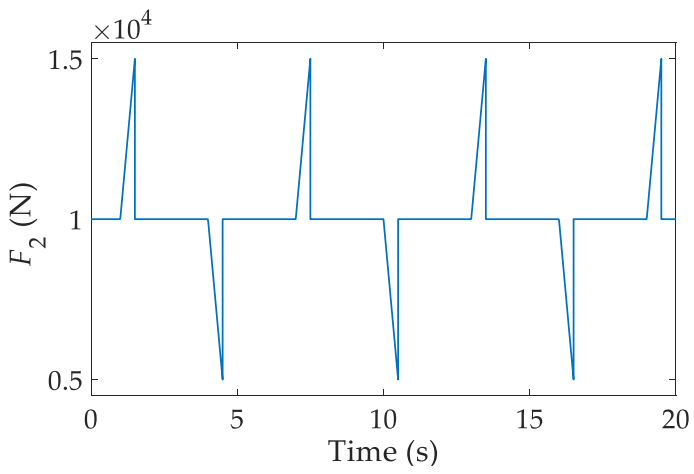

(b)

Figure 4. Signal diagram of the sum of load and disturbance $F$ : (a) load and disturbance $F_{1}$; (b) load and disturbance $F_{2}$.

\subsection{Simulation analysis}


A simple sinusoidal signal and a multi-frequency sinusoidal signal were created as reference inputs to the model. Under the condition that $Q$ is a positive definite matrix, appropriate parameters of controller are selected as $k_{1}=200, k_{2}=200, k_{3}=0.01, k_{4}=0.01, c_{1}=0.5, c_{2}=0.5, \lambda_{1}=1, \lambda_{2}=1, \lambda_{3}=0.5, \lambda_{4}=0.3$, $\lambda_{5}=100$.

\subsubsection{Simple sinusoidal signal}

A simple sinusoidal single, $y_{\mathrm{d}}=0.15 \sin \left(\frac{\pi}{5} t-\frac{\pi}{2}\right)+0.15$, is used as the reference signal, and the tracking performances of the ABSMC and the general PID system are compared through simulation.

Without disturbance, the position tracking is shown in Figure 5(a), and the position tracking error is shown in Figure 5(b).

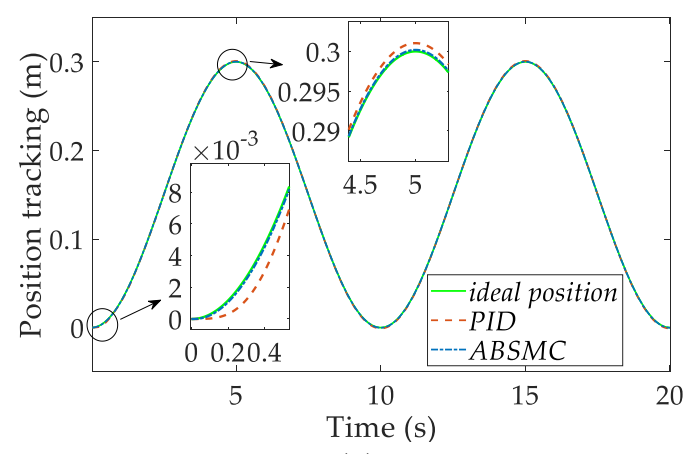

(a)

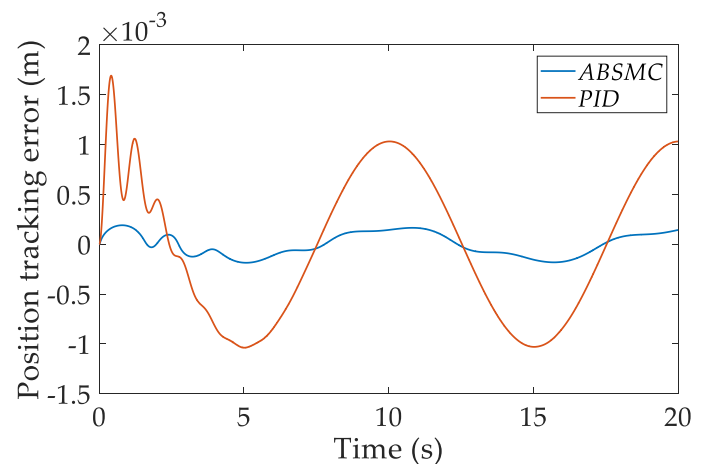

(b)

Figure 5. Simple sinusoidal responses of double-pump DDH using ABSMC and PID without disturbance: (a) position tracking without disturbance ; (b) tracking error without disturbance.

The simple sinusoidal curve was taken as the target displacement input the DDH system, and the output displacement and error were observed by adding different disturbance. The simulation responses of the DDH position tracking with $F_{1}$ is shown in Figure $6(\mathrm{a})$, and the position tracking error is show $n$ in Figure $6(\mathrm{~b})$. Similarly, the simulation response of position tracking with $F_{2}$ is shown in Figure 6(c), and the position tracking error results is shown in Figure 6(d).

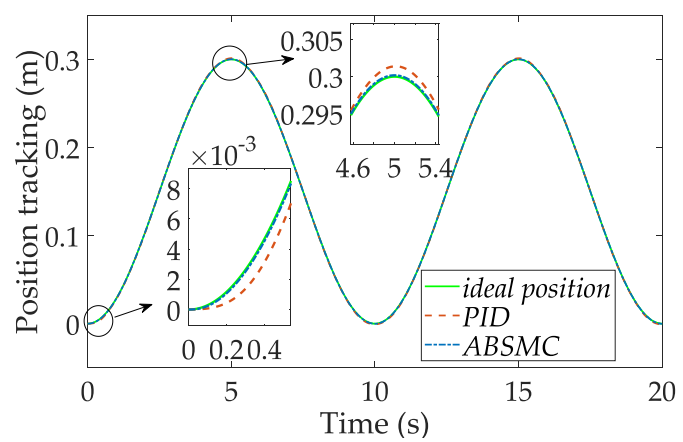

(a)

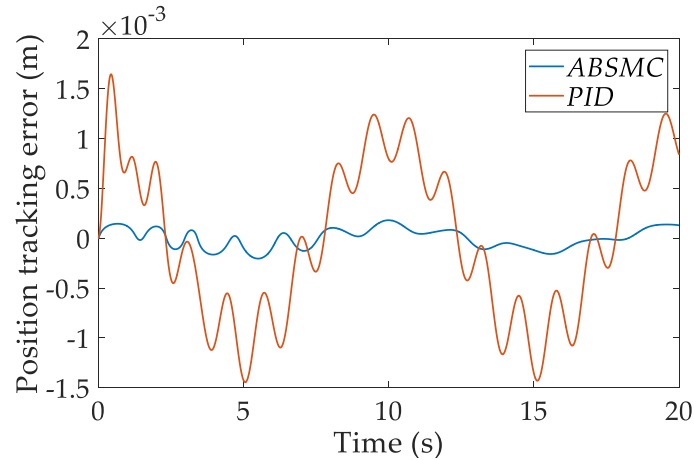

(b) 


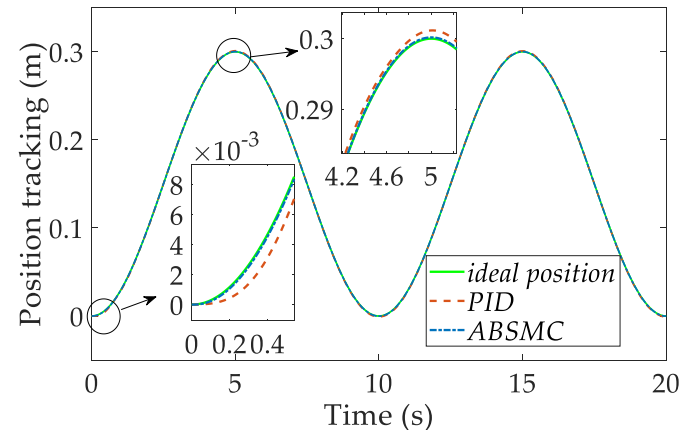

(c)

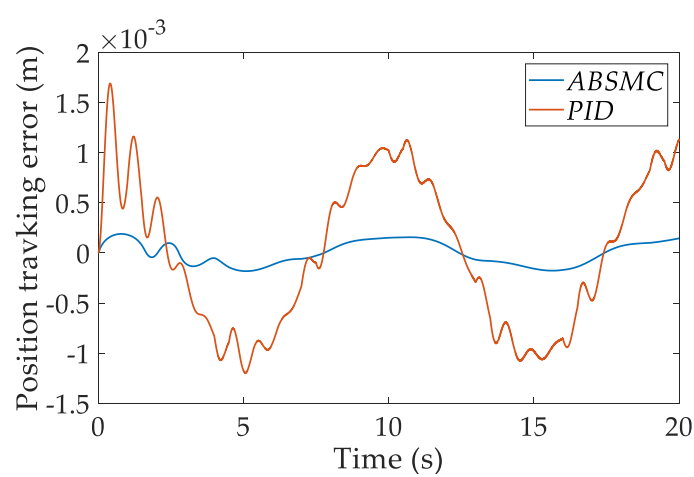

(d)

Figure 6. Simple sinusoidal response of DDH using ABSMC and PID with disturbance: (a) position tracking with $F_{1} ;(\mathrm{b})$ tracking error with $F_{1} ;(\mathrm{c})$ position tracking with $F_{2} ;\left(\right.$ d) tracking error with $F_{2}$.

From the simulation results, the ABSMC position tracking error is much smaller compared with the traditional PID control. Moreover, the response speed is faster than that of traditional PIDcontrol. With or without disturbance, the position tracking error of ABSMC basically remain unchanged, the error is small, and have strong anti-disturbance capability, good robustness.

\subsubsection{Multi-frequency sinusoidal signal}

Then, taking $y_{\mathrm{d}}=0.05\left[\sin \left(\frac{2 \pi}{5} t-\frac{\pi}{2}\right)+\sin \left(\frac{\pi}{5} t-\frac{\pi}{2}\right)+\sin \left(\frac{\pi}{10} t-\frac{\pi}{2}\right)+\sin \left(\frac{2 \pi}{25} t-\frac{\pi}{2}\right)+4\right]$ as the reference signal, and the tracking performances of the ABSMC and the general PID system are compared through simulation.

Without disturbance, the simulation responses of position tracking are shown in Figure 7(a). The position tracking error is shown in Figure $7(\mathrm{~b})$.

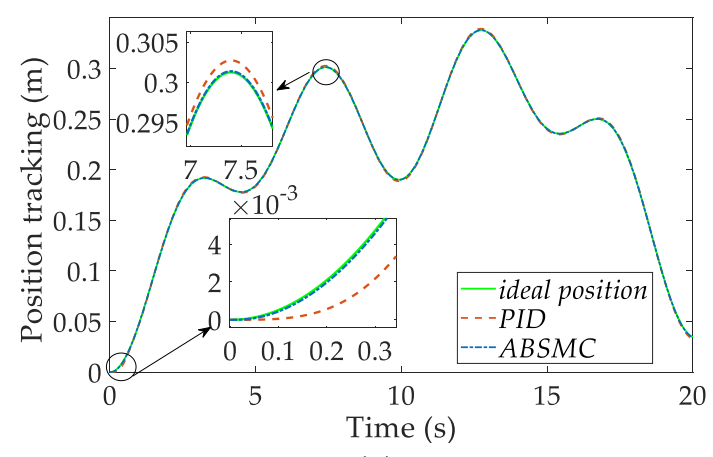

(a)

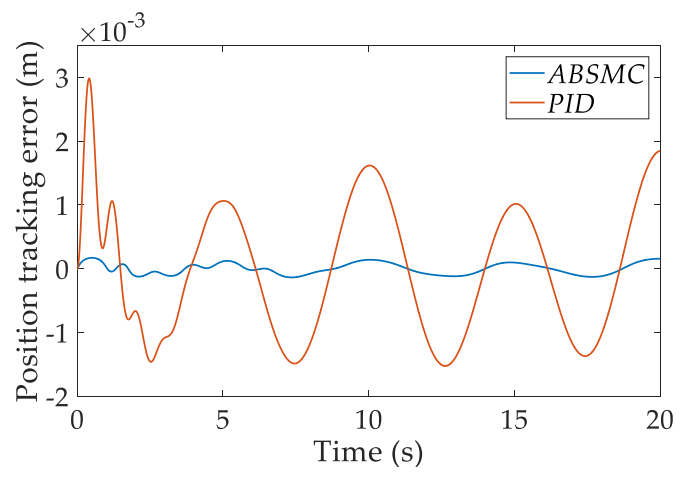

(b)

Figure 7. Multi-frequency sinusoidal re sponse of DDH using ABSMC and PID without disturbance: (a) position tracking without disturbance; (b) tracking error without disturbance.

The multi-frequency sinusoidal curve was taken as the target displacement input the DDH system, and the output displacement and error were observed by adding different disturbance. The simulation responses of DDH position tracking with $F_{1}$ is shown in Figure 8(a), and the position tracking error is show $n$ in Figure 8(b). Similarly, the simulation response of position tracking with $F_{2}$ is shown in Figure 8(c), and the position tracking error results is shown in Figure 8(d).

The simulation results show that when the system is uncertain, with or without disturbance, the $\mathrm{ABSMC}$ is stable in the position tracking of the $\mathrm{DDH}$, and the system output can track the position 
reference faster. Compared with the PID controller, the designed controller has small tracking error, fast response speed, better tracking performance, and good robustness.

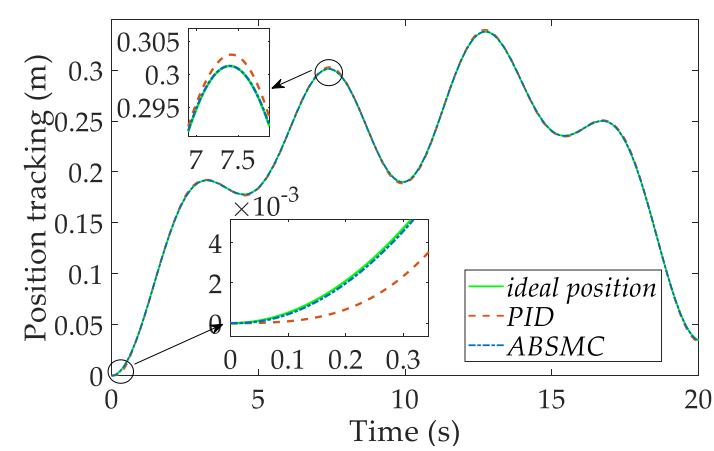

(a)

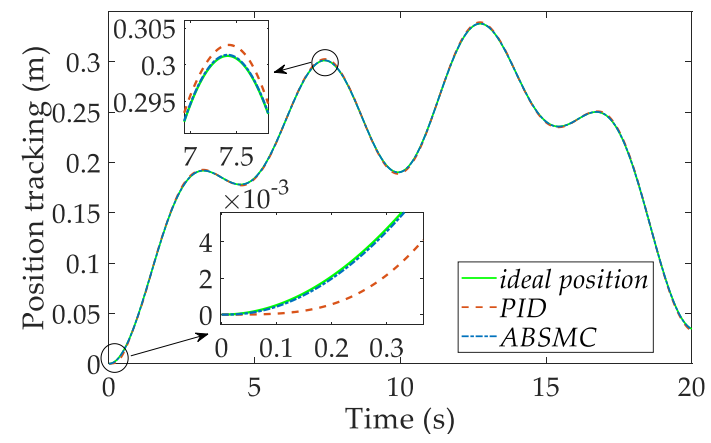

(c)

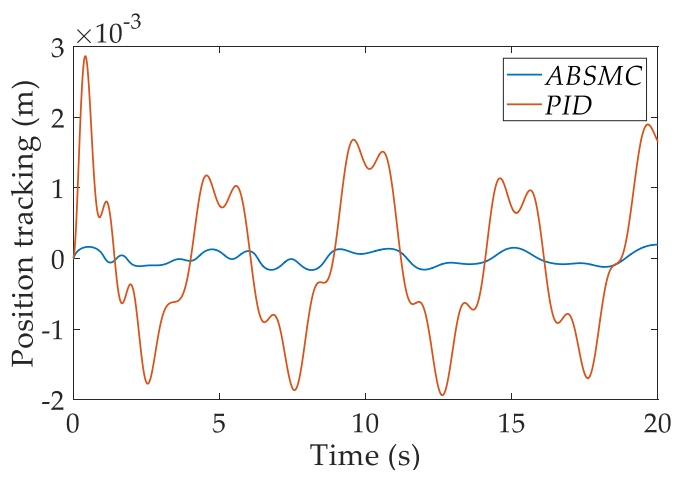

(b)

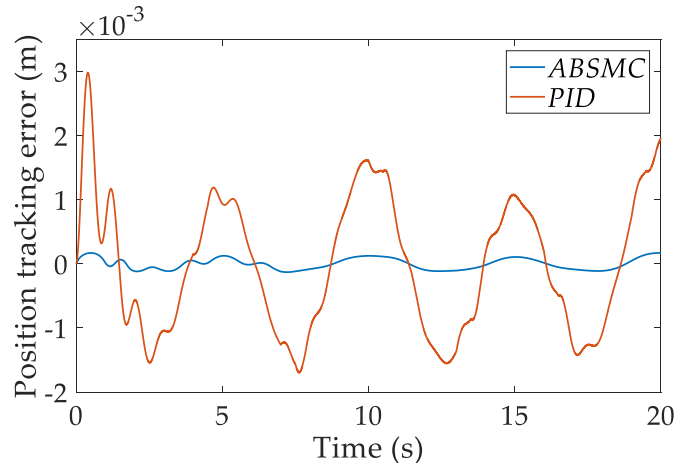

(d)

Figure 8. Multi-frequency sinusoidal response of the DDH using ABSMC and PID with disturbance: (a) position tracking with $F_{1}$; (b) tracking error with $F_{1}$; (c) position tracking with $F_{2}$; (d) tracking error with $F_{2}$.

\section{Conclusions}

In this paper, a controller adopting ABSMC was proposed for the double-pump DDH with unknown parameters, using an adaptive backstepping algorithm based on a linearized system model. According to the Lyapunov analysis and design, the ABSMC employs the appropriate parameters adaptive law to ensure the stability of the closed-loop system and the boundness of the parameters, which is supported by a theoretical proving. The proposed controller can address the problems of nonlinear characteristics, parameter uncertainty and uncertainty coefficient ahead of control input. A model was built, including the DDH, and ABSMC controller. Simulations were performed using two types of reference signal. The simulation results show that ABSMC can track the position accurately under varying load disturbances, no matter with simple or complex position reference. This control method can effectively address the problem that the designed control quantity and adaptivelaw are nested with each other due to the uncertainty coefficient ahead of the control input. It can effectively overcome the influence of the system's nonlinearity and parameter uncertainty, fast and accurate tracking and has strong robustness to parameter changes.

Although the simulation results show that ABSMC method can improve the position control accuracy of double-pump DDH, it lacks experimental data for comparison and verification. Therefore, the following research should establish a test bench and conduct experiment to validate the simulation results. In addition, the ABSMC method designed in this paper adopts the conventional 
design process, that is, the first two steps are backstepping control, and then combined with sliding mode control, and an adaptivelaw is given. Next, the design process can be innovated.

Acknowledgments: This work was supported by the Science Founda tion for Young Scholars of Fujian Province (No.2018J05099), the Scientific Research Fund (No. GY-Z15096), Fujian Haiyuan Composite Materials Technology Co., Ltd. and the Public Service Platform for Technical Innovation of Machine Tool Industry in Fujian University of Technology.

Author Contributions: Conceptualization, S.Z., and T.C. and F.D.; methodology, S.Z. and T.C.; software, T.C.; investigation, S.Z. and T.C.; writing - original draft preparation, S.Z. and T.C.; writing - review and editing, F.D.; supervision, S.Z.; project a dministration, F.D.; funding acquisition, F.D. All authors have read and agreed to the published version of the manuscript.

Conflicts of Interest: The authors declare no conflict of interest.

\section{Abbreviations}

The following abbreviations are used in this manuscript:

EHA: Electro-hydraulic actuator.

DDH: Direct driven hydraulics.

ABSMC: Adaptive backstepping sliding mode control.

PID: Proportional-integral-diffe rential.

\section{References}

1. Ketelsen, S.; Padovani, D.; Andersen, T.O.; Ebbesen, M.K.; Schmidt, L. Classifica tion and Review of PumpControlled Diffe rential Cylinder Drives. Energies 2019, 12, 1293.

2. Kai, Z. Research on the Cha racteristics of Position Control Based on Variable Speed Pump ControlSystem. Master's The sis, Shanghai Jiao Tong University, Shanghai,China, 2017.

3. Grabbel, J.; Ivantysynova, M. An Investigation of Swash Plate Control Concepts for Displacement Controlled Actua tors. International Journal of Fluid Power 2005, 6, (2), 19-36.

4. Niraula, A.; Zhang, S.; Minav, T.; Pietola, M. Effect of ZonalHydraulics on Energy Consumption and Boom Structure of a Micro-Excavator. Energies (Basel) 2018, 11, (8), 2088.

5. Agostini, T.; Negri, V.D.; Minav, T.; Pietola, M. Effect of Energy Recovery on Efficiency in ElectroHydrostatic Closed System for Differential Actua tor. Actuators 2020, 9, (1), 12.

6. Altare, G.; Vacca, A. A Design Solution for Efficient and Compact Electro-hydraulic Actuators. Procedia Engineering 2015, 106, 8-16.

7. Fu, S.; Wang, L.; Lin, T. Control of electric drive powe rtrain based on va riable speed control in cons truction machine ry. Automation in Construction 2020, 119, 103281.

8. Lin, T.; Lin, Y.; Ren, H.; Chen, H.; Chen, Q.; Li, Z. Development and key technologies of pure electric construction machinery. Renewable and Sustainable Energy Reviews 2020, 132, 110080.

9. Izzuddin, N.H.; Faudzi, A.M.A.; Johari, M.R.; Osman, K. System identification and pre dictive functional control for electro-hydraulic actua tor system. In IEEE International Symposium on Robotics \& Intelligent Sensors, IEEE: Langkawi, Malaysia, 2015; pp 138-143.

10. Tony Thomas, A.; Parameshwaran, R.; Sathiyavathi, S.; Vimala Starbino, A. Improved Position Tracking Performance of ElectroHydraulic Actua tor Using PID and Sliding Mode Controller. Iete Journal of Research 2019, 1-13.

11. Yao, J.; Deng, W. Active Disturbance Rejection Adaptive Control of Hydraulic Servo Systems. IEEE Transactions on Industrial Electronics 2017, 64, (10), 8023-8032.

12. Guo, K.; Wei, J.; Tian, Q. Nonline ar a da ptive position tra cking of an electro-hydraulic actuator. Proceedings of the Institution of Mechanical Engineers Part C Journal of Mechanical Engineering Science 2015, 229, (17), 32523265.

13. Alemu, A.E.; Fu, Y. In Sliding mode control of electro-hydrostatic actuator based on extended state observer, 
2017, 2017; IEEE: 2017; pp. 758-763.

14. Qi, H.; Liu, S.; Yang, R.; Yu, Y. Research on new intelligent pump controlbased on sliding mode va riable structure control. In 2017 IEEE International Conference on Mechatronics and Automation (ICMA), Takamatsu, Japan, 2017.

15. Miao, Z.; Cao, Y. Application of fuzzy PID controller for valveless hydraulic system driven by PMSM. Journal of Physics: Conference Series 2019, 1168, 022010.

16. Guo, J.; Ye, C.; Wu, G. Simulation and Research on Position Servo Control System of Opposite Vertex Hydraulic Cylinder based on Fuzzy Neural Network. In 2019 IEEE International Conference on Mechatronics and Automation(ICMA), Tianjin, China, 2019; pp 1139-1143.

17. Dong, H.; Gao, L.; Shen, P.; Li, X.; Lu, Y.; Dai, W. An interval type-2 fuzzy logic controller design method for hydraulic actuators of a human-like robot by using improved drone squadron optimization. International Journal of Advanced Robotic Systems 2019, 16, (6), 172988141989155.

18. Zad, H.S.; Ulasyar, A.; Zohaib, A. Robust Model Predictive Position Control of Direct Drive ElectroHydraulic Servo System. In 2016 International Conference on Intelligent Systems Engineering (ICISE), Islamabad, Pakistan, 2016.

19. Zhou, H.; Lao, L.; Chen, Y.; Yang, H. Discre te-time sliding mode control with an input filter for an electrohydraulic actua tor. IET Control Theory E Applications 2017, 11, (9), 1333-1340.

20. Järf, A. Flow Compensation Using Hydraulic Accumulator in Direct Driven Hydraulic Differential Cylinder Application and Effects on Energy Efficiency. Master's The sis, Aalto University, Es poo, Finland, 2016.

(C) 2020 by the authors; licensee MDPI, Basel, Switzerland. This article is an open access article dis tribute $\mathrm{d}$ under the terms and conditions of the Creative Commons by Attribution (CC-BY) license (http://creativecommons.org/licenses/by/4.0/). 